\title{
Erratum
}

\section{Torelli theorems for singularities}

\section{Yakov Karpishpan}

Department of Mathematics, The John Hopkins University, Baltimore, MS 21218, USA

Invent. Math. 100, 97-141(1990)

The paper of the title contains several errors.

1. The MHS on the Milnor fiber is an invariant of the map $f$, not of the singularity $f^{-1}(0)$. The attempt in the paper to define a variation of this MHS for deformations of $f^{-1}(0)$ runs into certain difficulties. To avoid this problem one must replace deformations of $f^{-1}(0)$ by unfoldings of $f$. In particular, we must work with the miniversal unfolding in place of the miniversal deformation, i.e. $T^{1} \cong Q^{f}$, while $M^{f}$ should be ignored. Also, the Gauss-Manin system of an unfolding with the base $S$ is defined on $S \times \mathbf{C}$ and not on $S$.

2. In the formula given in the Remark at the end of (1.11) $N$ must be replaced by $\log _{\tau} T_{s}+N$ (cf. (1.5)).

3. The Proposition and its Corollary in (5.8) are wrong: M. Saito [M. S7, (3.9)] gave an example of a $\mu=$ constant unfolding $f_{r}$ and forms $\omega_{1}, \ldots, \omega_{\mu}$ constituting a good basis for $f$ but not for $f_{r}, r \neq 0$. Therefore Theorem (5.3) is false: the set $\mathscr{K}$, as defined in (5.3), depends on the choice of a good basis, and, in general, one can only assert that $\mathscr{K} \subseteq D_{\mu}$.

Nevertheless, the "conditional" infinitesimal Torelli theorem (4.1) is true when restated for unfoldings. To confirm it one may follow [V-Ch], as was mentioned in (4.3), and use relative forms $\tilde{\omega} \in \Omega_{\mathscr{X} / \Delta_{r}}^{n+1}$ (or $\left.\tilde{\omega} \in \Omega_{\mathscr{X} / A}^{n+1}\right)$ in place of the absolute forms $\omega \in \Omega_{X}^{n+1}$ (cf. the definition of $\mathscr{H}^{\prime \prime}$ in (8.2)).

4. The analysis of "iso-Hodge" deformations in $\S 8$ is not valid in the non-quasihomogeneous case. In general, along a trajectory of a logarithmic vector field in $D_{\mu}$ the Hodge filtration on the vanishing cohomology may not stay constant. Also, the Euler vector field $E$ will, in general, have a non-zero constant term $w \in T^{1}$ corresponding to $f \in Q^{f}$ under the isomorphism $Q^{f} \cong T^{1}$. The set $\mathscr{M}$ introduced in (8.5) may not equal $D_{\tau} \cap D_{\mu}$, and in general there seems to be no good way to restate the local Torelli Theorem (8.5) for unfoldings.

In the quasi-homogeneous case

$$
\mathscr{M}=D_{\tau} \cap D_{\mu}=\left\{\text { the degree zero part of } S \text { under the } \mathbf{C}^{*} \text {-action }\right\},
$$


and here the statements of Theorem (8.5) and Lemma (8.14), on which it is based, can be seen directly, without the theory of logarithmic vector fields.

5. The statement on primitive forms established in (8.12) under some conditions has been shown in full generality by Morihiko Saito [M.S7, (3.11)]. I also wish to mention that in [M.S 2, 6, 7] he developed a different notion of the period map, via Brieskorn lattices, which induces our period map after taking $G r_{V}$, and which is finite-to-one on all of $D_{\mu}$.

I thank Morihiko Saito, Claus Hertling, and the referees for pointing out my mistakes.

\section{Added references}

[M.S6] Saito, M.: On the structure of Brieskorn lattices, Ann. Inst. Fourier 39, 27-72 (1989) (revised version of [M.S4])

[M.S7] Saito, M.: Period Mapping via Brieskorn Modules. Bull. Soc. Math. France 119, 141-171 (1991) 